\title{
99mTc-labeled boronic acid derivatives for SPECT myocardial perfusion imaging: Improved version 2.0
}

\author{
Raymond Taillefer, MD, FRCP, ABNM ${ }^{\mathrm{a}}$ \\ a Department of Nuclear Medicine, Hôpital du Haut-Richelieu, CISSS de la Montérégie Centre, \\ Saint-Jean-sur-Richelieu, QC, Canada
}

Received Mar 6, 2020; accepted Mar 6, 2020

doi: $10.1007 / \mathrm{s} 12350-020-02137-w$

See related article, pp. 2687-2696

According to several observers, the late 1980s and early 1990 s are considered to be the « golden age » of nuclear cardiology, regarding the myocardial perfusion imaging (MPI) field with Single Photon Emission Computed Tomography (SPECT). Effectively, within a few years, three differents 99mTc-labeled MPI agents have been approved by the Food and Drug Administration (FDA) for clinical use : 99mTc-sestamibi, 99mTc-teboroxime and $99 \mathrm{mTc}$-tetrofosmin. ${ }^{1-4}$ In the early 1990s several others 99mTc-MPI agents have been the subjects of basic, animal and clinical researches such as $99 \mathrm{mTc}$-Furifosmin and $99 \mathrm{mTc}-\mathrm{N}-\mathrm{NOEt} .{ }^{5,6}$ However, these two agents have not been approved. At the same time, PET myocardial perfusion tracers started to be progressively introduced in clinical practice. Although during the last 30 years several studies have been performed in order to synthesize new SPECT MPI agents, no significant potential radiotracer has emerged, so that 99mTc-sestamibi and 99mTc-Tetrofosmin are still used in almost every SPECT MPI clinical studies around the world, with the exception of 201Thallium for more specific indications.

Like 99mTc-Sestamibi, 99mTc-Teboroxime became commercially available in december 1990, when they both have been almost simultaneously approved by the FDA. 99mTc-Teboroxime, is chemically very

Reprint requests: Raymond Taillefer, MD, FRCP, ABNM, Department of Nuclear Medicine, Hôpital du Haut-Richelieu, CISSS de la Montérégie Centre, 920 Boulevard du Séminaire Nord, Saint-Jeansur-Richelieu, QC J3A 1B7, Canada; rtaillefer@hotmail.com

J Nucl Cardiol 2021;28:2697-9.

$1071-3581 / \$ 34.00$

Copyright (C) 2020 American Society of Nuclear Cardiology. different from 99mTc-sestamibi or 99mTc-Tetrofosmin. It has a smaller molecular size than $99 \mathrm{mTc}$-sestamibi but is larger than 201Thallium. A neutral and highly lipophilic compound (99mTc-sestamibi is a cationic complex), $99 \mathrm{mTc}$-Teboroxime is a member of the boronic acid adducts of technetium dioxime complexes (BATO). These complexes are neutral seven coordinate technetium vicinal dioxime complexes that have a boron group at one end of the molecule. $99 \mathrm{mTc}$-Teboroxime is the generic name for (bis(1,2-cyclohexanedione dioximato(1-)-0)-(1,2-cyclohexane-dione-ioximato(2-)-0) methyl borato(2-)-N, $\left.\mathrm{N}^{\prime}, \mathrm{N}^{\prime \prime}, \mathrm{N}^{\prime \prime \prime}, \mathrm{N}^{\prime \prime \prime \prime}, \mathrm{N}^{\prime \prime \prime \prime \prime}\right)$-chloro-technetium, also referred as SQ30217 (developmental name) or cadiotec (trademark name from Squibb Diagnostics).

Human biodistribution also differs from that of 99mTc-sestamibi. ${ }^{7-10}$ After intravenous administration at rest, 99mTc-teboroxime diffuses rapidly across the phospholipid cell membrane due to its neutral and highly lipophilic characteristics. Blood and lung activity clears within 1 to 2 minutes after the injection. Blood clearance is rapid with only $9.5 \%$ of the dose remaining in the circulation 15 minutes after the injection. The liver, which is the major route of elimination, shows a low activity initially, but the hepatic uptake increases over time with peak activity starting about 5 minutes after injection. The hepatic half-time differs from that of 99mTc-sestamibi because it is approximately 1 to 1.5 hours, suggesting that the mechanisms of uptake and excretion may also differ. Myocardial uptake of $99 \mathrm{mTc}-$ teboroxime is very rapid, with excellent myocardial visualization at 1 to 2 minutes after injection. The myocardial clearance, however, is also very rapid and biexponential, with half-times of 2 minutes $(68 \%)$ and 78 minutes $(32 \%)$. 99mTc-Teboroxime has a high firstpass myocardial extraction fraction and shows a better linear relationship between its myocardial uptake and myocardial blood flow (MBF) than that of 99mTc-sestamibi and 99mTc-Tetrofosmin. 
Table 1. Criteria for the "ideal" spect myocardial perfusion imaging radiotracer

1. High myocardial uptake with no or minimal myocardial redistribution

2. A high and stable target-to-background ratio with low uptake in the adjacent organs (lungs, liver, stomach) during the image acquisition period

3. Linear relationship between radiotracer myocardial uptake and coronary blood flow: $<5 \mathrm{~mL} / \mathrm{min} / \mathrm{gr}$ (level seen with pharmacological vasodilation). This requires a high first-pass myocardial extraction fraction and a subsequent very rapid blood clearance

4. A physical half-life that allow enough time for adequate image acquisition (and repeated acquisition if necessary) with both treadmill stress test and pharmacological intervention

5. Quantification of absolute myocardial blood flow, enabling identification of diffuse, multivessel, or balanced CAD

6. Appropriate radiation dosimetry and safety profile

Although approved by the FDA at the same time than 99mTc-Sestamibi, 99mTc-Teboroxime has quickly become far less commonly used in clinical practice. The above mentionned peculiar pharmacokinetic properties of $99 \mathrm{mTc}$-Teboroxime have challenged the users of this radiotracer, especially to find a clinically useful and optimal imaging protocol. Because it takes between 1 and 2 minutes for the 99mTc-Teboroxime blood pool activity to clear, the hepatic uptake peaks at about 5 to 6 minutes after the injection and progressively increases overtime, and the myocardial wash-out is very rapid, there is a narrow time window for optimal MPI with 99mTc-Teboroxime. Dynamic SPECT with threeheaded SPECT system has been proposed as an alternative to the standard systems but the number of this type of SPECT cameras was rather limited in clinical practice. ${ }^{11}$ Fast image acquisition protocols or dedicated cardiac SPECT gamma cameras with high count efficiency (CZT) would have been ideal for this radiotracer but since these were not available at that time, $99 \mathrm{mTc}$ teboroxime rapidly disappeared from the clinical pratice.

In this current issue of the Journal, $\mathrm{Xi}$ and collaborators $^{12}$ present the continuation of their initial study performed in Sprague-Dawley rats using a newly developed MPI radiotracer, 99mTc-3SPboroxime, $99 \mathrm{mTcCl}(\mathrm{CDO})(\mathrm{CDOH}) 2 \mathrm{~B}-3 \mathrm{SP}) 3 \mathrm{SP}-\mathrm{B}(\mathrm{OH}) 2=3-$

(methylsulfonyl)pyridineboronic acid, another member of the boronic acid adduct $99 \mathrm{mTc}$ (III) complexes. This time they studied the myocardial uptake, the heart-toliver ratio and the $\mathrm{K} 1$ and $\mathrm{K} 2$ values for compartment model in normal and acute myocardial infarction swines. Three different radiotracers were compared: 99mTc3SPboroxime. 99mTc-Teboroxime and 99mTc-Sestamibi. Their results showed that the initial uptake was comparable to that of $99 \mathrm{mTc}$-Teboroxime but significantly higher than that of $99 \mathrm{mTc}$-sestamibi. The heartto-liver ratio of $99 \mathrm{mTc}$-SPboroxime was higher than that of $99 \mathrm{mTc}$-Teboroxime at 15 minutes post-injection. The
K1 value of 99mTc-3SPboroxime was significantly much higher than that of $99 \mathrm{mTc}$-Sestamibi (suggesting its higher first-pass myocardial extraction fraction) and the $\mathrm{K} 2$ values were significantly lower than that of 99mTc-Teboroxime (both at rest and after dypiridamole administration, indicating a longer $99 \mathrm{mTc}-3$ SPboroxime myocardial retention time). These two characteristics are of prime importance in order to detect the proper extent and severity of myocardial ischemia. The authors were able to obtain high quality SPECT images over the first 15 minutes after the injection, using a cadmium zinc telluride (CZT) SPECT camera. Therefore, an initial increased myocardial uptake coupled to a prolonged myocardial retention of $99 \mathrm{mTc}-3 \mathrm{SPboroxime}$ in comparison to $99 \mathrm{mTc}$-Teboroxime, offer a significant advantage of enabling a wider image time acquisition window for MPI and offering a more accurate MBF quantitation with dynamic SPECT, which is much more difficult to obtain with $99 \mathrm{mTc}$-Teboroxime.

One legitimate question arises: why do we need a new 99mTc-MPI radiotracer since those available in clinical practice for almost the last 30 years have been shown to provide satisfactory results? Table 1 summarizes the most important criteria for the ideal SPECT MPI radiotracer. ${ }^{13}$ Although $99 \mathrm{mTc}-$ Sestamibi and 99mTc-Tetrofosmin shows many of the required characteristics, they suffer from a less than ideal relationship between radiotracer myocardial uptake and $\mathrm{MBF}$, with the « roll-off » phenomenon reported at high flow rates, especially seen when using pharmacologic intervention. Because of this sub-optimal non-linear relation between radiotracer uptake and $\mathrm{MBF}$, proper physiological compensation is required for quantification of the absolute coronary blood flow and flow reserve with SPECT, which can be sometimes inaccurate and difficult to realize.

Obviously, although these preliminary results are very interesting, more studies will be needed in order to 
validate the potential use of $99 \mathrm{mTc}$-3SPboroxime in MBF quantitation with direct comparison with gold standard MBF measurements such as perfusion PET imaging or microspheres and, of course, biodistribution data obtained in humans. Furthermore, one must not forget lessons learned from the past experiences: results obtained from animal studies cannot always be automatically and exactly extrapolated to humans. There are various examples in the past, especially with the isonitriles family (99mTc-CPI, 99mTc-TBI or 99mTc-RP42) where the animal studies showed great results but human biodistribution data were disappointing, showing either more increased lung or liver uptake or lower myocardial extraction, limiting their potential widespread clinical applications. $^{14-16}$

Nonetheless, a new 99mTc-labeled MPI radiotracer showing better myocardial uptake and retention with a better relationship between myocardial uptake and MBF, especially at high flow rates, would be more than welcome. This could be refreshing after 30 years of using the same SPECT MPI radiotracers. Furthermore, this could help, particularly with the new imaging softwares and CZT gamma cameras, to increase the role of nuclear cardiology, especially to fulfill its potential role in accurately quantifying regional MBF with SPECT imaging and increasing its diagnostic accuracy.

\section{Disclosure}

Raymond Taillefer declares he has no financial conflict of interest.

\section{References}

1. Taillefer R, Tamaki N. Technetium-99m sestamibi. In: New Radiotracers in cardiac imaging: Principles and applications. Appleton and Lange, Stamdord; 1999. pp. 1-48.
2. Beller GA, Watson DD. Physiological basis of myocardial perfusion imging with the technetium agents. Semin Nucl Med 1991;21:173-81.

3. Dahlberg ST, Leppo JA. Myocardial kinetics of radiolabeled perfusion agents: Basis for perfusion imaging. J Nucl Cardiol 1994;1:189-97.

4. Jones S, Hendel RC. Technetium-99m Tetrofosmin: A new myocardial perfusion agent. J Nucl Med Technol 1993;21:191-5.

5. Gerson MC, Lukes J, Deutsch EA, et al. Comparison of imaging properties of technetium 99m Q12 and technetium Q3 in humans. J Nucl Cardiol 1995;2:224-30.

6. Fagret D, Marie PY, Brunotte F, et al. Myocardial perfusion imaging with technetium-99m-NOET: Comparison with thallium and coronary angiography. J Nucl Med 1995;36:936-43.

7. Narra RK, Feld T, Nunn AD. Absorbed radiation dose to humans from technetium-99m-teboroxime. J Nucl Med 1992;33:88-93.

8. Pieri PL, Strauss HW. Advances in myocardial perfusion imaging: 99mTc-teboroxime. J Nucl Biol Med 1992;36:22-8.

9. Taillefer R, Lambert R, Essiambre R, et al. Comparison between thallium-201, technetium-99m-sestamibi and technetium-99m-teboroxime planar myocardial perfusion imaging in detection of coronary artery disease. J Nucl Med 1992;33:1091-8.

10. Weinstein H, Dahlberg ST, McSherry B, et al. Rapid redistribution of teboroxime. Am J Cardiol 1993;71:848-52.

11. Nakajima K, Taki J, Bunko H, et al. Dynamic acquisition with a three-headed SPECT system: Application to technetium 99mSQ30217 myocardial imaging. J Nucl Med 1991;32:1273-7.

12. Xi, XY, Wang L, Hsu B, et al. $99 \mathrm{mTc}-3$ SPboroxime: A neutral $99 \mathrm{mTc}(\mathrm{III})$ radiotracer with high heart uptake and long myocardial retention. J Nucl Cardiol Current issue.

13. Dilsizian V, Taillefer R. Journey in evolution of nuclear cardiology. State-of-the art paper. Will there be another quantum leap with the F-18-labeled myocardial perfusion tracers. J Am Coll Cardiol 2012;5:1269-84.

14. Deutsch E, Ketring AR, Libson K, et al. The Noah's ark experiment: Species dependent biodistributions of cationic complexes. Int J Radiol Appl Instrum 1989;16:191-232.

15. Holman BL, Jones AG, et al. A new Tc-99m-labelled imaging agent, hexakis (T-butyl-isonitrile)-technetium(I) (Tc-99m-TBI): Initial experience in the human. J Nucl Med 1984;25:1350-5.

16. Liu XJ, Wang X, Liu Y, et al. Clinical evaluation of 99mTc-CPI myocardial perfusion imaging. Eur J Nucl Med 1989;15:277-9.

Publisher's Note Springer Nature remains neutral with regard to jurisdictional claims in published maps and institutional affiliations. 